\title{
A Fuzzy Logic Controller to Increase Fault Ride-Through Capability of Variable Speed Wind Turbines
}

\author{
Geev Mokryani, Pierluigi Siano, Antonio Piccolo, and Vito Calderaro \\ Department of Industrial Engineering, University of Salerno, Salerno, 84084 Fisciano, Italy \\ Correspondence should be addressed to Pierluigi Siano, psiano@unisa.it
}

Received 4 October 2011; Revised 21 December 2011; Accepted 10 January 2012

Academic Editor: Anyong Qing

Copyright $\odot 2012$ Geev Mokryani et al. This is an open access article distributed under the Creative Commons Attribution License, which permits unrestricted use, distribution, and reproduction in any medium, provided the original work is properly cited.

A fuzzy controller for improving Fault Ride-Through (FRT) capability of Variable Speed Wind Turbines (WTs) equipped with Doubly Fed Induction Generator (DFIG) is presented. The controller is designed in order to compensate the voltage at the Point of Common Coupling (PCC) by regulating the reactive and active power generated by WTs. The performances of the controller are evaluated in some case studies considering a different number of wind farms in different locations. Simulations, carried out on a real 37-bus Italian weak distribution system, confirmed that the proposed controller can enhance the FRT capability in many cases.

\section{Introduction}

Wind turbines (WTs) are typically located in remote and rural areas. In these areas, the feeders are long and operated at a medium voltage level characterized by a high $\mathrm{R} / \mathrm{X}$ ratio and unbalanced voltage situations. Furthermore, weak grids are usually referred to have a "low short-circuit level" or "low fault level." In a weak network a change in the real and reactive power can cause a considerable change in the voltage. The impact relies on the strength of the network and the output power of the WTs [1]. Integration of WTs into weak grids can cause the steady-state voltage level to go outside of its acceptable limit. Therefore, it can limit the exploitation of wind energy resources. Another constraint is related to the effect of the power generated by WTs on the voltage quality. Voltage level limitations and accurate control systems are required to control the voltage variations as well as to improve the voltage quality [2], and variable speed WTs can be used as reactive power sources for voltage control.

In recent times, many researches have been carried out in this field. A proportional-integral- (PI-) based control algorithm to control the reactive power produced by WTs has been proposed in [3]. In [4], the authors have proposed a mathematical model of the Doubly Fed Induction Generator (DFIG) for the analysis of active and reactive power performances of a wind farm (WF). In [5], the relation between reactive and active power to maintain the DFIG's operation inside the maximum rotor and stator currents has been studied. In [6], the authors have proposed a fuzzy controller to manage the operation of a Flywheel Energy Storage System (ESS) connected to the DC bus.

Recently, the penetration of WTs into the grids increased, and the performance of the WTs under faults has became an important issue, especially for DFIGs. Several grid codes prescribed, in fact, that WTs should remain connected to the network during and after faults. The ability of WTs to stay connected to the grid during fault and voltage variations is defined as Fault Ride-Through (FRT) capability [7]. One of the common FRT capability improvement solutions is to set up a crowbar circuit across the rotor terminals [8]. Another common method is to insert an ESS [9], such as batteries or super capacitors at the DC bus inside the rotor side converter (RSC). A control strategy to improve FRT capability by using Flexible AC Transmission System (FACTS) devices and ESS has been proposed in [10]. In [11], the authors have proposed a new feed-forward transient current control (FFTCC) applied to RSC for improving the FRT capability. In [12], a fuzzy controller to manage the rotor speed oscillations and the DC-link voltage variations of the DFIG has been proposed.

In this paper, a fuzzy controller for improving FRT capability of variable-speed WTs in wind farms is presented. 
The controller is designed in order to compensate the voltage at the point of common coupling (PCC) by controlling the reactive and active power generated by WFs. In previous research works, other authors have investigated the FRT capability improvement only considering voltage sag, while in this paper the voltage swell effect is also considered to investigate FRT capability improvement according to Danish grid code. The novelty of the proposed fuzzy controller consists in considering active and reactive power regulation simultaneously. In particular, if during a voltage swell, the absorbed reactive power is not adequate to lower the voltage at the PCC within its statutory limits, the reference signal for the active power production is decreased by the fuzzy controller. In this case the WT will not generate the maximum active power according to its power coefficient, but this will determine two positive effects of the voltage regulation at the PCC: firstly, due to the limited size of the power converters of DFIGs, the active power reduction will allow increasing the maximum reactive power that can be absorbed by WTs; moreover, in medium-voltage weak networks with long feeders characterized by a high $\mathrm{R} / \mathrm{X}$ ratio, the active power decrease can also increase the voltage drop on the feeders thus contributing to lower the voltage at the PCC.

The performance of the controller is analyzed for both voltage sags and swells considering different load values. Different locations have been assumed for the WFs, and simulations have been carried out on a real 37-bus weak distribution network. The Danish grid code for the voltage levels below $100 \mathrm{kV}$ is used in order to evaluate the FRT capability improvement.

The paper is organized as follows: grid code requirements and FRT capability are presented in Section 2. The WT generator system and the fuzzy controller designing are discussed in Sections 3 and 4, respectively. Case studies and simulation results are discussed in Section 5.

\section{Grid Code Requirements and FRT Capability}

In recent years, the increasing penetration of WTs into power systems has led power system operators to develop new grid code requirements for WTs in many countries. These requirements impose WTs operators to deal with some aspects such as FRT capability, reactive power control, and voltage control. This implies that the WTs connection into a distribution system requires coordination with voltage and reactive power control. Several countries have provided different grid codes depending on their system characteristics and operation standards such as the code from E.ON Netz Germany, Denmark, Belgium, the UK, Spain, the Netherlands, USA, and Canadian TSO Hydro-Quebec [13]. The Nordic grid code from Nordel [14] specifies the technical requirements that new WTs should have in order to be connected at the transmission network and provide acceptable safe operation and reliability. Belgium grid code [15], provided by the Belgian TSO, Elia, applies to the grids with the voltage levels $30-70 \mathrm{kV}$ and $150-380 \mathrm{kV}$. This code discriminates different kinds of voltage disturbances. German grid code from E.ON Netz Germany [16] applies to the grids with the voltage levels of $110 \mathrm{kV}, 220 \mathrm{kV}$, and $380 \mathrm{kV}$ [17].

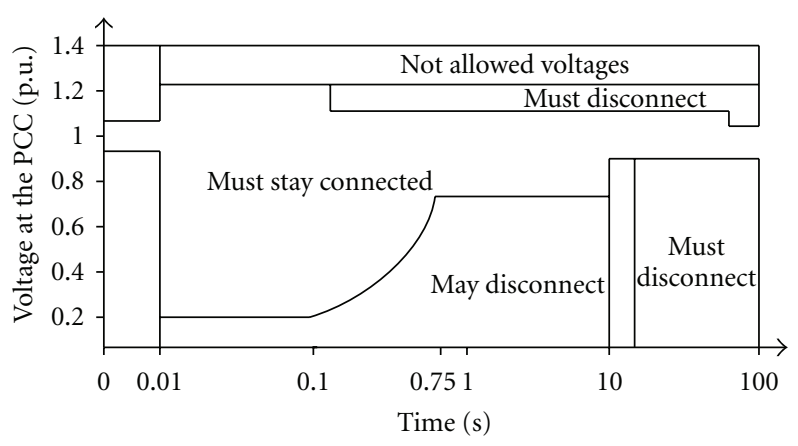

FIGURE 1: FRT requirements of the Danish grid code for grids below $100 \mathrm{kV}$ [17].

Denmark grid code [17] relates to the WTs connected to the grids with the voltages below $100 \mathrm{kV}$. Figure 1 illustrates the FRT requirements of the Danish grid code. Apart from the FRT requirements shown in Figure 1, WFs must disconnect if the voltage increases above 1.2 p.u.

\section{Description of Fuzzy Controller}

Due to nonlinearity of power system and linearization problems, the control of variable wind speed WTs could not perform correctly with conventional control methods. For example, a Proportional Integral (PI) controller design requires identifying the WT transfer function, the linear model of the network, and defining an accurate tuning process.

The use of a fuzzy controller can overcome these problems and counterbalance the nonlinearities as well as time variances of the system under control of which an accurate model is not required. In the case of WTs control, for example, inaccurate aerodynamic calculations, tolerance in mounting the turbine, dirt or ice on blades, time-varying aerodynamic parameters, and other unpredictable parameter variations can make fuzzy logic control preferable if compared to conventional control methods [18].

The proposed fuzzy controller presents the many advantages if compared to a PI controller [19, 20]: (1) it is easy in obtaining variable gains depending on the error; (2) it is simple in solving problems affected by uncertain models, (3) it gives fast convergence, (4) it is parameter insensitive and accepts noisy and inaccurate signals.

Considering that, for a given combination of the active and the reactive power, the maximum reactive power that can be injected/absorbed by a WT depends on both the power converter size and the generated active power, the maximum reactive power that the WT can inject/absorb can be increased by decreasing the generated active power according to the following formula:

$$
|Q|=\sqrt{S^{2}-P^{2}}
$$

where $S$ is the power converter size, given as maximum apparent power, $P$ and $Q$ are the generated active and reactive power, respectively. The proposed controller is designed in 


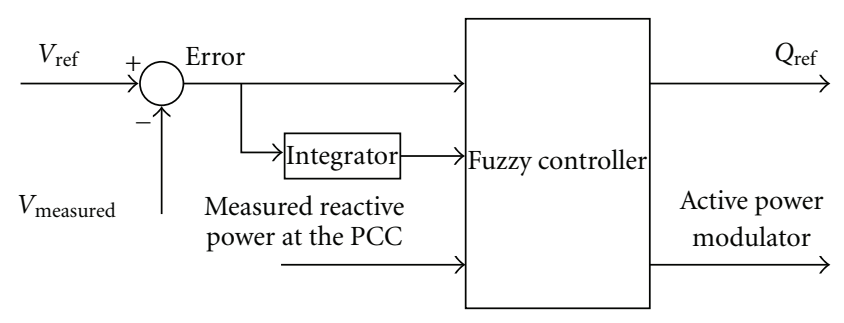

FIGURE 2: Fuzzy controller.

order to compensate the voltage at the PCC by injecting/absorbing the reactive power generated by both of grid side converter (GSC) and RSC as well as by regulating the active power modulator used to decrease the generated active power in the case of voltage swell.

In particular, when the absorbed reactive power reaches $80 \%$ of its maximum value and the measured voltage exceeds the reference value, the active power is decreased by the fuzzy controller in order to increase the maximum reactive power that the WT can absorb. Moreover, it is used in combination with a protection system for disconnecting the WTs from the grid when the controller is unable to compensate the voltage variations.

The fuzzy controller, as shown in Figure 2, presents three inputs: the error, the integral of error, and the measured reactive power at the PCC and two outputs that are the reference values for both the reactive power reference signal $\left(Q_{\text {ref }}\right)$, varying in the range $[-11]$, and the reference signal for the active power modulator, varying in the range $\left[\begin{array}{ll}0 & 1\end{array}\right]$, both sent by the fuzzy controller to the WT local controllers.

The error is defined as the difference between the reference voltage $\left(V_{\text {ref }}\right)$ and the measured voltage at the PCC.

The third input, the reactive power measured at the PCC, is used in order to determine when the active power modulator is required to be regulated by the fuzzy controller.

The reactive power reference signal $\left(Q_{\mathrm{ref}}\right)$ is sent by the fuzzy controller to the WTs local controllers in order to inject/absorb reactive power. The active power modulator is the reference signal for the active power production and is sent by the fuzzy controller to the WTs local controllers in order to decrease the injected active power with the aim of increasing the absorbed reactive power according to (1).

The implementation of the fuzzy controller requires an adequate knowledge base and the ability to transform the latter in a set of fuzzy rules. The knowledge base has been coded in a set of rules consisting of linguistic statements linking a finite number of conditions with a finite number of conclusions. Such a knowledge can be collected and delivered by human experts and expressed by a finite number $(r=$ $1,2, \ldots n)$ of heuristic Multiple Input Single Output (MISO) fuzzy rules, written in the following form:

$$
\begin{aligned}
& R_{\mathrm{MISO}}^{(r)}: \operatorname{IF}\left(x \text { is } A_{i}^{(r)}\right),\left(y \text { is } B_{i}^{(r)}\right), \ldots,\left(z \text { is } C_{i}^{(r)}\right) \\
& \operatorname{THEN}\left(u \text { is } U_{j}^{(r)}\right)^{\prime \prime},
\end{aligned}
$$

where $A_{i}^{(r)}, B_{i}^{(r)}, \ldots, C_{i}^{(r)}$ are the values of linguistic variables (conditions) $x, y, \ldots, z$, defined in the universes of discourse: $X, Y, \ldots, Z$, respectively, and $U_{j}^{(r)}$ is the value of independent linguistic variable $u$ in the universe of discourse $U$.

Among all the parameters associated with a FLC, membership functions (MFs) have a dominant effect in changing its performance.

The type of MFs is frequently chosen to fit an expected input data distribution or clusters and can influence both the tracking accuracy and the execution time. Triangular, trapezoidal, and Gaussian membership functions are the common choice even if any convex shape can be adopted. Even though most researchers are inclined to design the input/output fuzzy membership sets using equal span mathematical functions, these do not always guarantee the best solution.

In the proposed approach, the selection of the best membership functions has been performed on the basis of a prior knowledge and on experimentation with the system and its dynamics. In particular, triangular and Gaussian membership functions have been compared. Moreover, in order to design a FLC, shrinking span MFs have been chosen: this guarantees smoother results with less oscillations, large and fast control actions when the system state is far from the set point, and moderate and slow adjustments when it is near to the set point. Thus, when the system is closer to its set point, the fuzzy MFs, for those specific linguistic terms, have narrower spans.

The fuzzy sets of the inputs and outputs assume the following names: NVB: Negative-Very-Big, NB: Negative-Big, NM: Negative-Medium, NS: Negative-Small, ZE: Zero, and so forth. Nine triangular membership functions have been selected for the inputs and outputs. The membership functions of inputs and outputs are shown in Figure 3.

Inference rules for the controller can be derived by the control surfaces and some of these rules are provided in Table 1. 86 rules are used in order to design the fuzzy controller. A Mamdani-based system architecture is realized; Max-Min and Centroid methods are used in the inference engine and defuzzification process, respectively.

Two control surfaces of the fuzzy controller are provided in Figure 4. According to Figure 4(a), in the case of voltage sag (error is positive) only $Q_{\text {ref }}$ is sent by the fuzzy controller to the local controllers of the WTs in order to inject reactive power while in the case of voltage swell (error is negative), when the $Q_{\text {ref }}$ is not enough to compensate the voltage swell effects, the active power modulator is sent by the fuzzy controller to the local controllers of WTs in order to decrease the active power, according to (1), and consequently increase the absorbed reactive power.

As evidenced from Figure 4(b), when the absorbed reactive power reaches $80 \%$ of its maximum value and the measured voltage exceeds the reference value, the active power is decreased by the fuzzy controller in order to increase the maximum reactive power that the WT can absorb. Moreover, it is used in combination with a protection system for disconnecting the WTs from the grid when the controller is unable to compensate the voltage variations. 


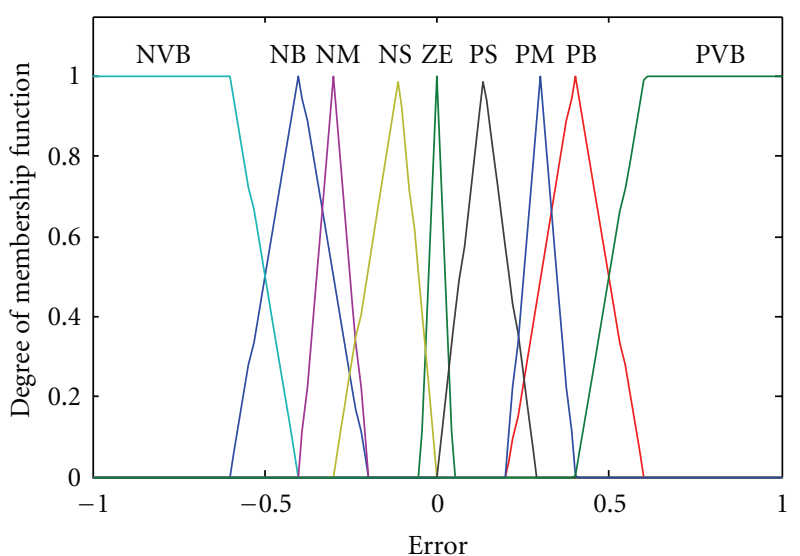

(a)

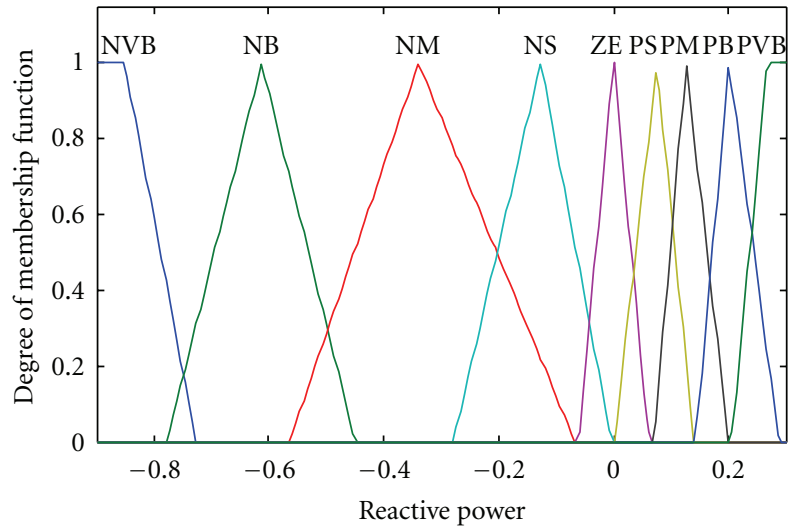

(c)

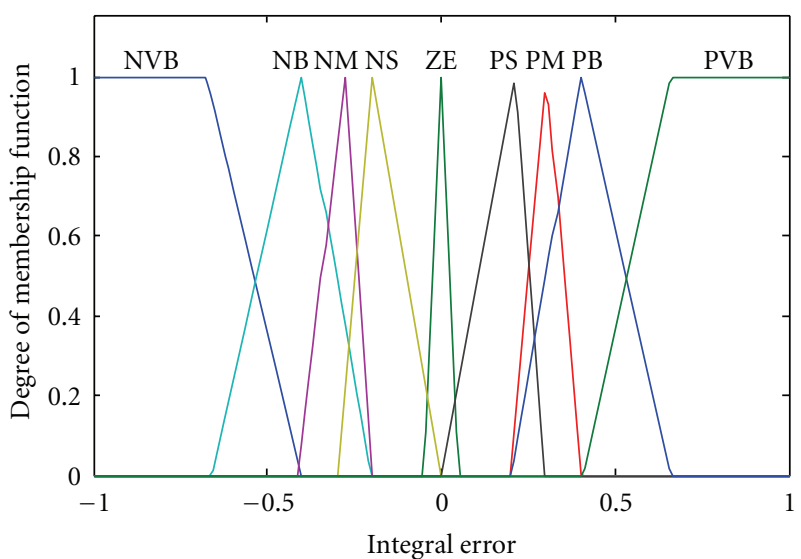

(b)

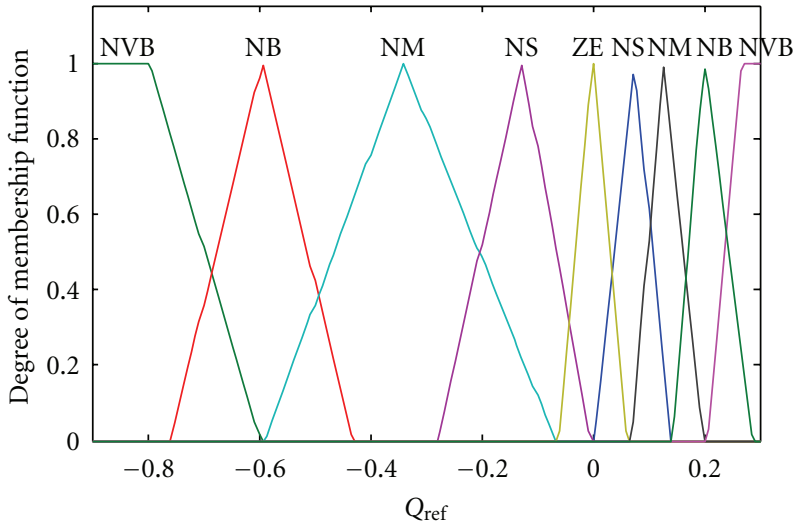

(d)

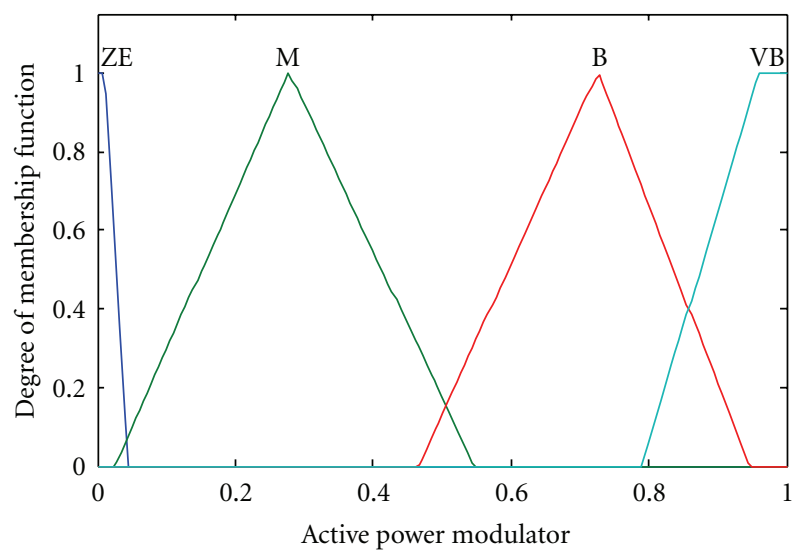

(e)

FIGURE 3: Membership functions of fuzzy controller. (a) Input signal error, (b) input signal integral error, (c) input signal reactive power measured at the PCC, (d) output reference reactive power, and (e) output active power modulator.

An easy understanding of the fuzzy controller can be summarized as in following considerations.

(1) If error is positive and integral of error is positive and reactive power measured at the PCC is positive, then reactive power should be injected by the WTs.

(2) If error is negative and integral of error is negative and reactive power measured at the PCC is not NVB, then reactive power should be absorbed by the WTs and the active power modulator is zero. Note that in this case, the absorbed reactive power is enough in order to decrease the voltage swell effects and there is no need to also regulate the active power.

(3) If error is negative and integral of error is negative and reactive power measured at the PCC is NVB, both reactive and active power are regulated in order to decrease the voltage swell effects. 
TABLE 1: Rules of fuzzy controller.

\begin{tabular}{cccccc}
\hline No. & Error & Integral error & Reactive power & Reference reactive power & Active power modulator \\
\hline 1 & NVB & $\sim$ PS & NVB & NB & B \\
2 & NVB & $\sim$ PVB & $\sim$ NVB & NVB & ZE \\
3 & NVB & NVB & $\sim$ NVB & NVB & VB \\
4 & NB & NS & $\sim$ NVB & NM & M \\
5 & NM & PM & $\sim$ NVB & NS & ZE \\
6 & NS & PS & $\sim$ NS & ZE & ZE \\
7 & ZE & ZE & ZE & PS & ZE \\
8 & PS & PVB & $\sim$ PS & PB & ZE \\
9 & PVB & NVB & $\sim \mathrm{PB}$ & & \\
\hline
\end{tabular}

$\sim$ Means NOT.

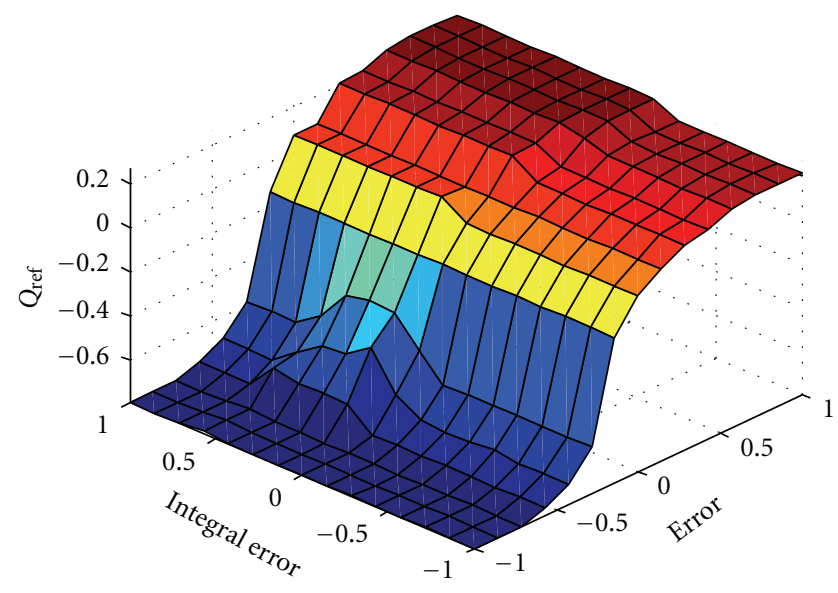

(a)

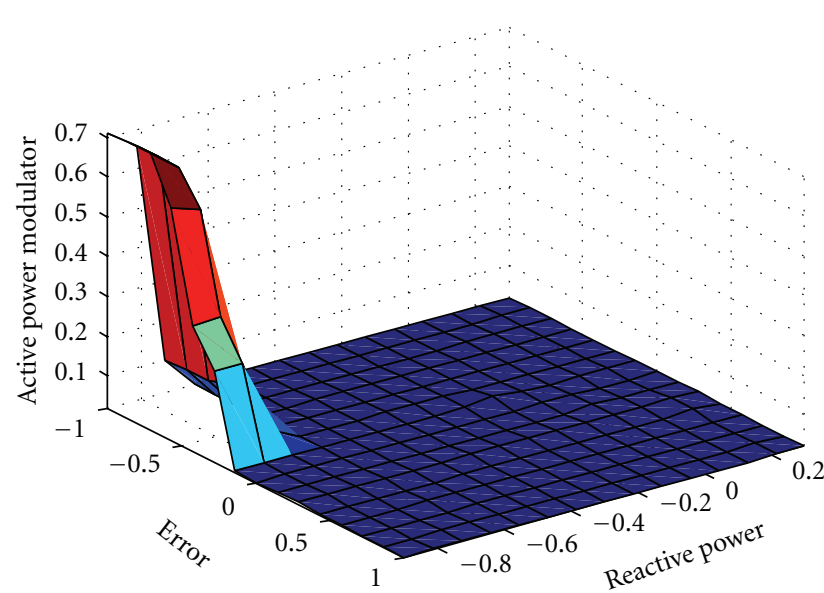

(b)

FIGURE 4: Fuzzy surface of controller for 1.5 MW WT.

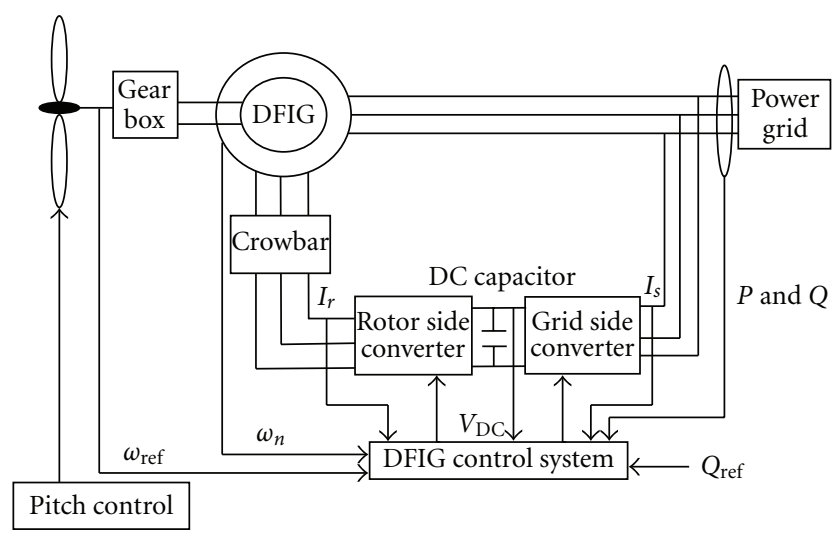

Figure 5: Configuration of DFIG-based WT connected to a grid.

\section{Wind Turbine Generator System}

A DFIG is a wound rotor induction generator with the stator directly connected to the grid and with the rotor connected to the network through a back-to-back converter. The schematic of DFIG-based WT is shown in Figure 5. The aim of the RSC is to control the active and reactive power on the grid autonomously, while the GSC has to maintain the DC-link voltage at a set value.

The relation between the wind speed and aerodynamic torque in a WT can be described by the following equation:

$$
\begin{aligned}
& P_{w}=\frac{1}{2} \rho \pi R^{2} V_{w}^{3} C_{p}(\theta, \lambda), \\
& T_{w}=\frac{1}{2} \rho \pi R^{2} V_{w}^{3} C_{p} \frac{(\theta, \lambda)}{\lambda},
\end{aligned}
$$

where $P_{w}$ and $T_{w}$ are the power and aerodynamic torque extracted from the wind in (W, N/m), respectively. $\rho$ is the air density $\left(\mathrm{kg} / \mathrm{m}^{3}\right), R$ is the wind turbine rotor radius $(\mathrm{m})$, $V_{w}$ is the equivalent wind speed $(\mathrm{m} / \mathrm{s}), \theta$ is the pitch angle wind turbine blade (deg.), $\lambda=\left(\omega_{\text {rot }} R\right) / V_{w}$ is the tip speed ratio, where $\omega_{\text {rot }}$ is the rotor speed of WT $(\mathrm{rad} / \mathrm{s})$ and $C_{P}$ is the aerodynamic efficiency of the rotor. $C_{P}$ can be expressed as a function of the tip speed ratio $(\lambda)$ and pitch angle $(\theta)$ by the following equation:

$$
C_{P}=0.22\left(\frac{116}{\beta}-0.4 \theta-5\right)^{e-12.5 / \beta},
$$


where $\beta$ is defined as follows:

$$
\beta=\frac{1}{(1 /(\lambda+0.08 \theta))-\left(0.035 /\left(\theta^{3}+1\right)\right)} .
$$

The induction generator converts the power captured by the WT into electrical power and transmits it into the grid. The AC/DC/AC converter consists of the RSC and the GSC. Both RSC and GSC are voltage source converters (VSCs) that use forced commutated power electronic devices to synthesize an AC voltage from a DC voltage source. A detailed description of the control systems for both the converters and the pitch angle command can be found in [21].

4.1. Capability Curve. The total active power of the DFIG fed into the grid is the sum of the stator and rotor active power.

$$
P_{T}=P_{S}+P_{R}
$$

taking into account that

$$
\begin{gathered}
P_{R}=-S P_{S}, \\
P_{T}=(1-S) P_{S},
\end{gathered}
$$

where $P_{T}$ is the total active power of the DFIG fed into the grid, $P_{S}$ is the stator active power, and $P_{R}$ is the rotor active power. The total reactive power fed into the grid is the sum of the reactive power of GSC and RSC. Usually, these inverters work with unity power factor, and consequently, the total reactive power will be equal to the reactive power of stator:

$$
Q_{T}=Q_{S}
$$

The active and reactive power of stator can be expressed as a function of the maximum allowable current of rotor and stator [22]:

$$
\begin{gathered}
P_{S}^{2}+Q_{S}^{2}=\left(3 U_{S}^{2} I_{S}^{2}\right) \\
P_{S}^{2}+\left(Q_{S}+3 \frac{U_{S}^{2}}{X_{S}}\right)^{2}=\left(3 \frac{X_{M}}{X_{S}} U_{S} I_{R}\right)^{2}
\end{gathered}
$$

Equation (10) represents a circumference centered equal to the stator rated apparent power. Equation (9) represents a circumference centered at $\left[-3\left(U_{S}^{2}\right) / X_{S}, 0\right]$. Substituting $(8)$ and (9) into (10) and (11) can be expressed as

$$
\begin{gathered}
\left(\frac{P_{T}}{1-S}\right)^{2}+Q_{T}^{2}=\left(3 U_{S} I_{S}\right)^{2} \\
\left(\frac{P_{T}}{1-S}\right)^{2}+\left(Q_{T}+3 \frac{U_{S}^{2}}{X_{S}}\right)^{2}=\left(3 \frac{X_{M}}{X_{S}} U_{S} I_{R}\right)^{2} .
\end{gathered}
$$

The DFIG capability limits according to (12) and (13) can be achieved by taking into consideration the stator and rotor maximum currents $I_{S \max }$ and $I_{R \max }$, respectively. However, the reactive power delivered to the grid is limited by constraints imposed by the converter and expressed as rotor current limits to avoid an excessive heating of converters,

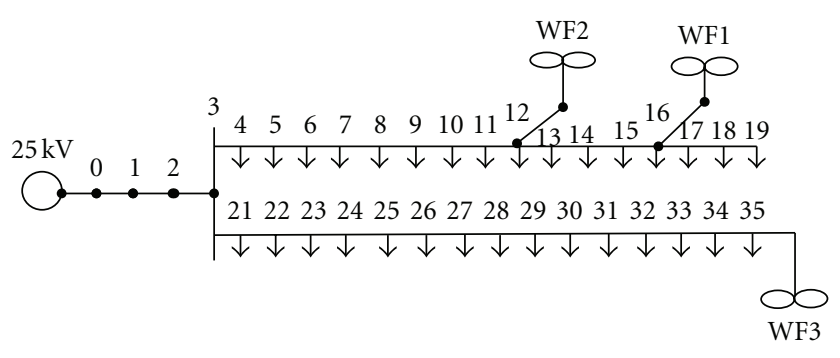

Figure 6: $25 \mathrm{kV}$ weak distribution system.

rotor slip-rings, and brushes. The stator reactive power limits $Q_{S, \lim }$ depend on the stator active power $P_{S}$, the stator voltage $U_{S}$, and the maximum rotor current $I_{R \max }$. It is expressed as follows:

$$
Q_{S, \lim }=-\frac{U_{S}^{2}}{X_{S}} \pm \sqrt{\left(\frac{X_{M}}{X_{S}} U_{S} I_{R \max }\right)^{2}-P_{S}^{2}}
$$

Equation (14) expresses the maximum reactive power that can be delivered to the grid.

\section{Case Study and Simulation Results}

A DFIG-based WT, shown in Figure 5, has been considered [23]. In order to test the proposed controller, three 9-MW WFs $(6 \times 1.5 \mathrm{MW}$ WTs $)$ connected to a real $25 \mathrm{kV}$ weak distribution system at buses 12, 16, and 35 are considered, as shown in Figure 6. The network is characterized by lines with high resistances and low $\mathrm{X} / \mathrm{R}$ ratios. The base value for the power and voltage are 9/0.9 MVA and $575 \mathrm{~V}$, respectively. According to the capability curve of the considered WFs, the limits of the reactive power that the WT can absorb or inject are 8 MVar and 2.50 MVar, respectively. Real wind data sets acquired by the Wind Engineering Research Field Laboratory [24] are considered. The wind speed time history consists of 17500 observations within 50-second interval with sampling rate $25 \mathrm{~Hz}$.

In order to test the FRT capability of the WFs endowed with the proposed fuzzy controller considering minimum and maximum load, four different scenarios are studied for both load conditions as follows, with a total of eight case studies:

(1) Case study 1: a 30\% voltage sag with a duration of 1 second starting at $t=5$, considering

(i) three WFs at buses 12,16 , and 35,

(ii) two WFs at buses 16 and 35.

(2) Case study 2: a 15\% voltage swell with a duration of 1 second starting at $t=5$, considering

(i) three WFs at buses 12,16 , and 35,

(ii) two WFs at buses 16 and 35.

5.1. Minimum Load. The total network loads are $18 \mathrm{MW}$ and 12.5 MVAr assumed as minimum load. 
TABLE 2: Results obtained with PI controller and without controller for all WFs.

\begin{tabular}{cccccrr}
\hline \multirow{2}{*}{ WF no. } & \multicolumn{2}{c}{ Proposed controller } & \multicolumn{2}{c}{ PI controller } & \multicolumn{2}{c}{ Without controller } \\
& Voltage (p.u.) & WF's situation & Voltage (p.u.) & WF's situation & Voltage (p.u.) & WT's situation \\
\hline 1 & 0.745 & Connected & 0.698 & Disconnected & 0.688 & Disconnected \\
2 & 0.740 & Connected & 0.695 & Disconnected & 0.685 & Disconnected \\
3 & 0.715 & Connected & 0.715 & Connected & 0.691 & Disconnected \\
\hline
\end{tabular}

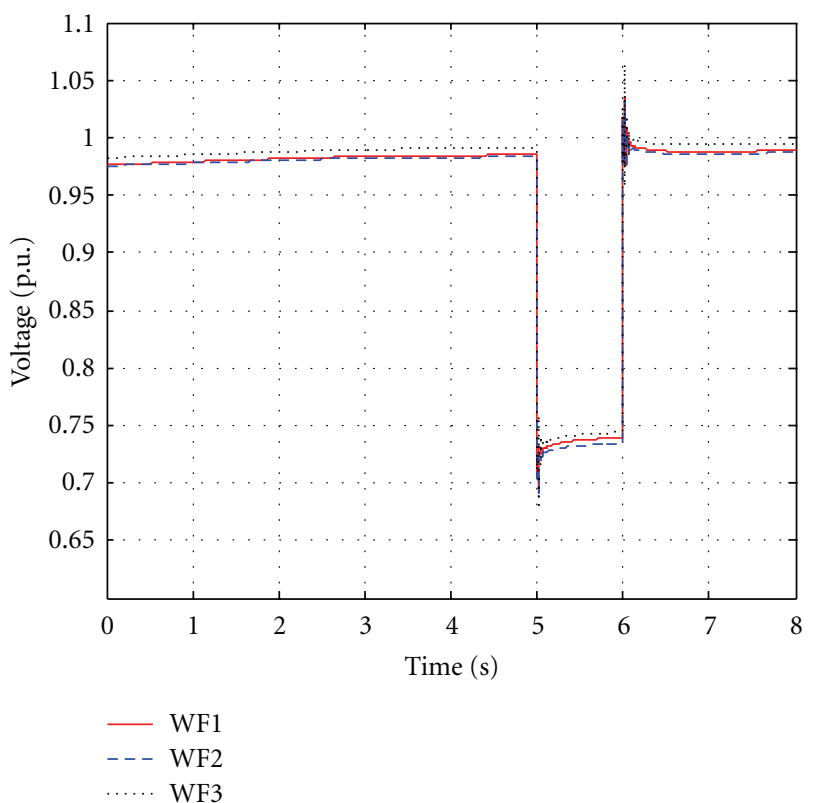

Figure 7: Voltage at the PCC.

5.1.1. Case Study A1 (Voltage Sag). (i) When the voltage drops by $30 \%$, each WF injects reactive power during the voltage sag in order to help in increasing the voltage to 0.725 , 0.730 , and 0.741 p.u. for WF2, WF1, and WF3, respectively (see Figure 7). The injected reactive power varies between about 2.25 MVAr for WF3 to about 2.40 MVAr for WF2 according to the voltages at the connection buses. According to Danish grid code, all WFs can successfully fulfill the FRT requirement and, consequently, remain connected to the grid.

In order to evaluate the effectiveness of the proposed controller, both of the cases without any controller and with a classical PI controller as designed in [25] are evaluated. The results are given in Table 2. It can be evidenced that with the fuzzy controller, all WFs can successfully fulfill the FRT requirement and remain connected to the grid while with the PI controller only WF3 can successfully fulfills the FRT requirement and remain connected to the grid while WF1 and WF2 disconnect. Moreover, without any controller, all WFs disconnect.

(ii) In this case, only WF1 and WF3 are considered. The reactive powers injected by the WFs increase if compared to the previous case and only WF3 can fulfill grid code requirements and remain connected to the grid (see Table 3 ).

5.1.2. Case Study A2 (Voltage Swell). (i) Considering three WFs during a $15 \%$ voltage swell, they absorb reactive power
TABle 3: Results obtained with proposed controller in the case of 2 WFs.

\begin{tabular}{ccc}
\hline WF no. & Voltage (p.u.) & WF's situation \\
\hline 1 & 0.690 & Disconnected \\
3 & 0.721 & Connected \\
\hline
\end{tabular}

in order to help lowering the voltage. The voltages, as shown in Figure 8(a), at buses 16, 12, and 35 are about 1.060, 1.070 , and 1.076 p.u., respectively. The absorbed reactive power varies between about 7 MVAr for WF1 to about $8 \mathrm{MVAr}$ for WF3, as shown in Figure 8(b), according to the voltages at the connection buses. Moreover, when the absorbed reactive power reaches $80 \%$ of its maximum value and is not enough for compensating voltage variations, the active power modulator is regulated in order to decrease the generated active power and consequently increase the reactive power absorbed by WFs (see Figure 8(c)). According to the capability curve of WTs and the limited size of the power converters, WFs cannot absorb reactive power more than 8 MVAr.

According to the fuzzy controller surface in Figure 4(b) and as shown in Figure 8(c), it can be observed that the active power modulator regulation starts at $t=5.2 \mathrm{~s}$. The combined regulation of both active and reactive power generated by WFs allows reducing the voltage swell effects. According to the Danish grid code, all WFs can successfully fulfill the FRT requirement and, consequently, remain connected to the grid. In order to assess the effectiveness of the proposed controller, the performance of the fuzzy controller is compared with that of a PI controller as designed in [25] and without any controller. As shown in Table 4, with the PI controller, WF1 and WF2 can successfully fulfill the FRT requirement and remain connected to the grid while WF3 disconnects; moreover, without any controller, all WFs are disconnected.

(ii) When considering only WF1 and WF3, it can be observed that the absorbed reactive powers by the WFs increase if compared to the previous case, while more active power is reduced.

For example, for WF3, the absorbed reactive power is about 7.8 MVAr in case (i) while a reduction of about $55 \%$ of active power is achieved. In case (ii), instead these values are about $8 \mathrm{MVAr}$ and $62 \%$, respectively. According to Table 5, both WF1 and WF3 can successfully fulfill the grid code requirements and remain connected to the grid. It can be concluded that, by increasing the number of WFs, their probability of remaining connected is increased.

5.2. Maximum Load. A total maximum load of $24 \mathrm{MW}$ and $15 \mathrm{MVAr}$ has been assumed. The same case studies 
TABLE 4: Results obtained with PI controller and without controller For All WFs.

\begin{tabular}{ccccccc}
\hline \multirow{2}{*}{ WF no. } & \multicolumn{2}{c}{ Proposed controller } & \multicolumn{2}{c}{ PI controller } & \multicolumn{2}{c}{ Without controller } \\
& Voltage (p.u.) & WF's situation & Voltage (p.u.) & WF's situation & Voltage (p.u.) & WT's situation \\
\hline 1 & 1.056 & Connected & 1.085 & Connected & 1.112 & Disconnected \\
2 & 1.059 & Connected & 1.090 & Connected & 1.115 & Disconnected \\
3 & 1.067 & Connected & 1.111 & Disconnected & 1.125 & Disconnected \\
\hline
\end{tabular}

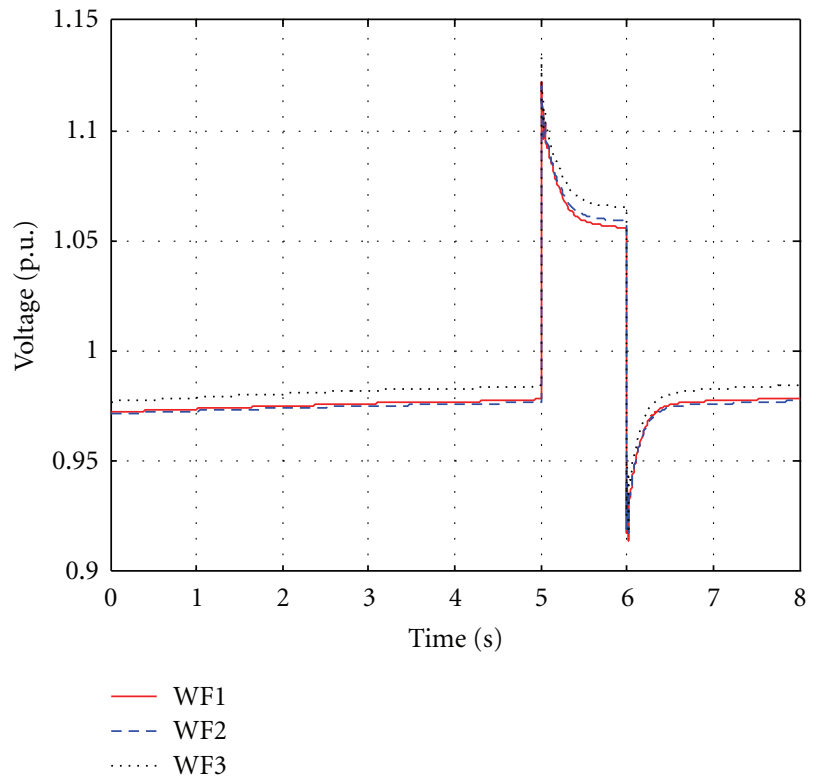

(a)

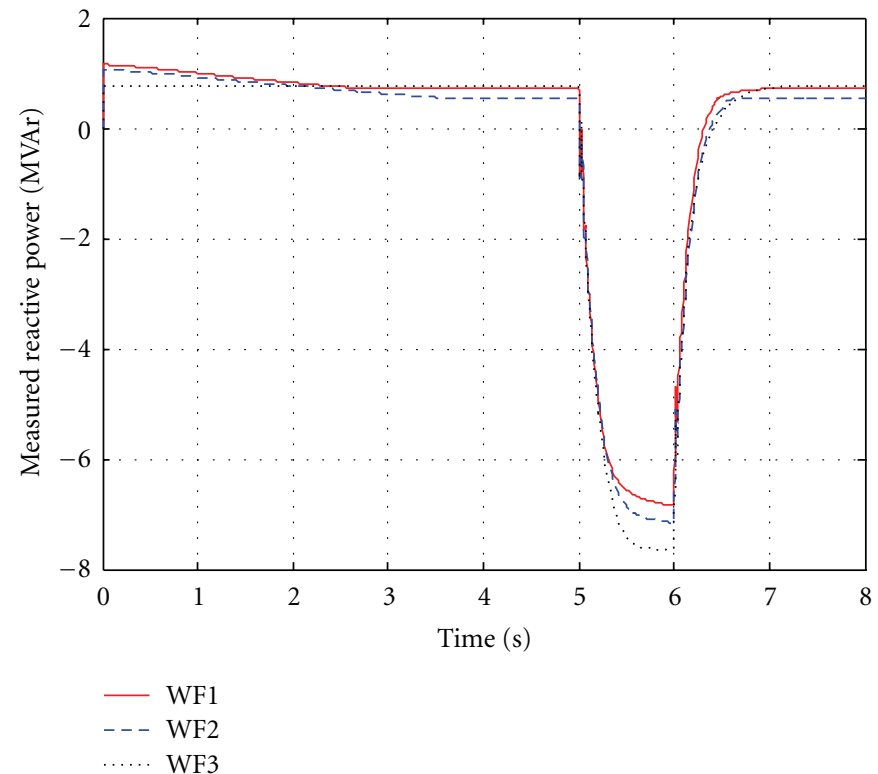

(b)

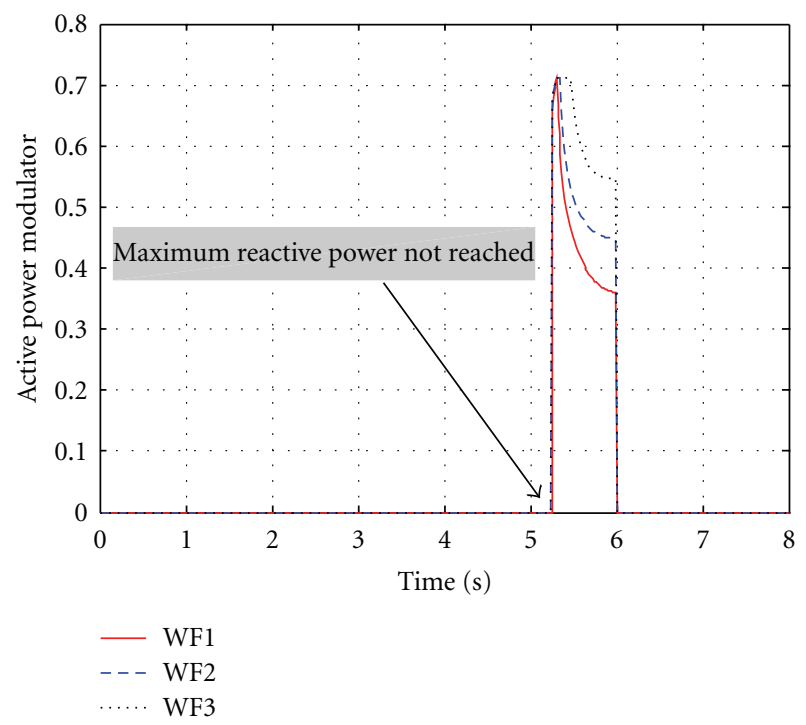

(c)

Figure 8: (a) Voltage, (b) measured reactive power, and (c) active power modulator at the PCC.

investigated in Section 5.1 for minimum load are also studied for maximum load.

5.2.1. Case Study B1 (Voltage Sag). (i) When the voltage drops by $30 \%$, each WF injects reactive power during the voltage sag in order to help in increasing the voltage. The injected reactive power varies between about 2.35 MVAr for WF3 to about 2.50 MVAr for WF2 according to the voltages at the PCC. In correspondence with maximum load, the voltage at the PCC decreases and the reactive powers 


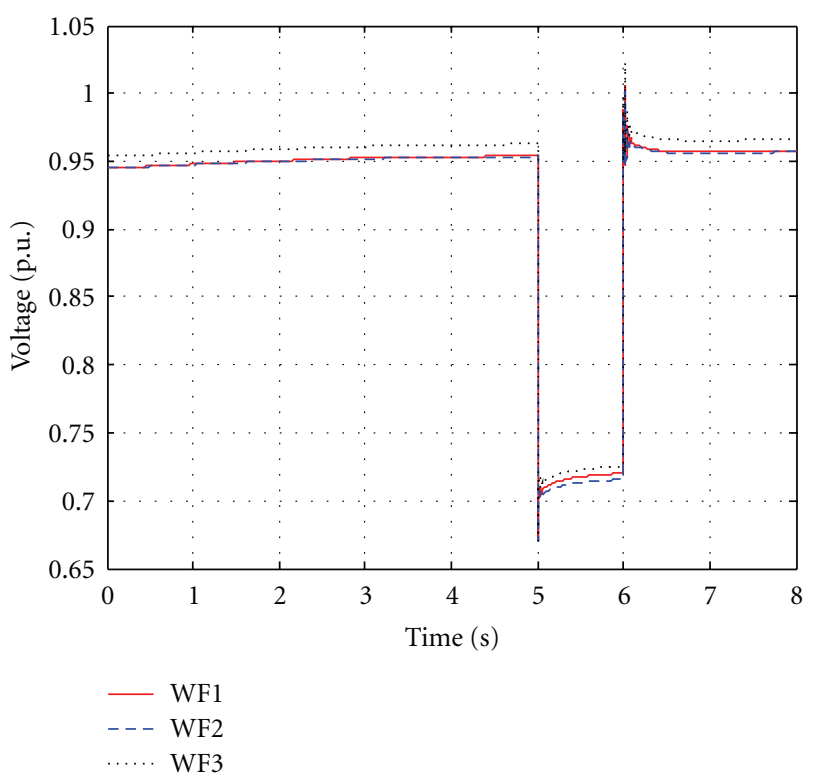

Figure 9: Voltage at the PCC.

TABLE 5: Results obtained with proposed controller in the case of 2 WFs.

\begin{tabular}{ccc}
\hline WF no. & Voltage (p.u.) & WF's situation \\
\hline 1 & 1.071 & Connected \\
3 & 1.062 & Connected \\
\hline
\end{tabular}

TABLE 6: Results obtained with proposed controller in the case of 2 WFs.

\begin{tabular}{ccc}
\hline WF no. & Voltage (p.u.) & WF's situation \\
\hline 1 & 0.684 & Disconnected \\
3 & 0.705 & Connected \\
\hline
\end{tabular}

injected by WFs increase if compared to the case of minimum load. As shown in Figure 9, all WFs can fulfill the grid code requirements and can remain connected to the grid.

(ii) By decreasing the number of WFs connected to the grid the injected reactive powers increase. According to Table 6, WF3 can fulfill the grid, code requirement and remain connected to the grid while WF1 disconnects from the grid.

5.2.2. Case Study B2 (Voltage Swell). (i) Considering three WFs during a $15 \%$ voltage swell, the absorbed reactive power varies between about 6 MVAr for WF1 to about 7 MVAr for WF3. A reduction of about $28 \%$ and $46 \%$ of active power is achieved for WF1 and WF3, respectively. According to the Danish grid code, all WFs can successfully fulfill the FRT requirements and, consequently, remain connected to the grid.

(ii) In this case, both WF1 and WF3 can fulfill the grid code requirement and remain connected to grid.

\section{Conclusion}

In this paper, a fuzzy controller for improving the FRT capability of variable speed WTs is proposed. The controller is designed in order to compensate the voltage-sags and swells by regulating both the active and reactive power generated by WFs. The performances of the proposed controller are evaluated in several case studies considering a 37-bus Italian weak distribution network with WFs in different locations. Simulation results evidenced that the proposed controller can improve WTs' FRT capability in medium-voltage weak networks in many cases, thus improving wind energy exploitation.

\section{References}

[1] I. M. de Alegría, J. Andreu, J. L. Martín, P. Ibañez, J. L. Villate, and H. Camblong, "Connection requirements for wind farms: a survey on technical requierements and regulation," Renewable and Sustainable Energy Reviews, vol. 11, no. 8, pp. 18581872, 2007.

[2] J. O. G. Tande, "Exploitation of wind-energy resources in proximity to weak electric grids," Applied Energy, vol. 65, no. $1-4$, pp. 395-401, 2000.

[3] G. Tapia, A. Tapia, and J. X. Ostolaza, "Proportional-integral regulator-based approach to wind farm reactive power management for secondary voltage control," IEEE Transactions on Energy Conversion, vol. 22, no. 2, pp. 488-498, 2007.

[4] G. Tapia, A. Tapia, and J. X. Ostolaza, "Two alternative modeling approaches for the evaluation of wind farm active and reactive power performances," IEEE Transactions on Energy Conversion, vol. 21, no. 4, pp. 909-920, 2006.

[5] W. Gao, G. Wang, and J. Ning, "Development of low voltage ride-through control strategy for wind power generation using real time digital simulator," in Proceedings of the IEEE/PES Power Systems Conference and Exposition (PSCE '09), pp. 1-6, March 2009.

[6] L. Jerbi, L. Krichen, and A. Ouali, "A fuzzy logic supervisor for active and reactive power control of a variable speed wind energy conversion system associated to a flywheel storage system," Electric Power Systems Research, vol. 79, no. 6, pp. 919-925, 2009.

[7] M. Tsili and S. Papathanassiou, "A review of grid code technical requirements for wind farms," IET Renewable Power Generation, vol. 3, no. 3, pp. 308-332, 2009.

[8] S. M. Muyeen, R. Takahashi, T. Murata et al., "Low voltage ride through capability enhancement of wind turbine generator system during network disturbance," IET Renewable Power Generation, vol. 3, no. 1, pp. 65-74, 2009.

[9] J. Liang, W. Qiao, and R. G. Harley, "Feed-forward transient current control for low-voltage ride-through enhancement of DFIG wind turbines," IEEE Transactions on Energy Conversion, vol. 25, no. 3, pp. 836-843, 2010.

[10] M. Rahimi and M. Parniani, "Grid-fault ride-through analysis and control of wind turbines with doubly fed induction generators," Electric Power Systems Research, vol. 80, no. 2, pp. 184-195, 2010.

[11] H Karimi-Davijani, A. Sheikholeslami, H. Livani, and M. Karimi-Davijani, "Fuzzy logic control of doubly fed induction generator wind turbine," World Applied Sciences Journal, vol. 6, no. 4, pp. 499-508, 2009.

[12] F. Iov, A. Hansen, P. Soerensen, and N. Cutululis, "Mapping of grid faults and grid codes," Technical Report of the Research 
Project 'Grid Fault and Design Basis for Wind Turbine', Riso National Laboratory, Roskilde, Denmark, 2007.

[13] Nordel, "Nordic grid code," January 2007.

[14] Grid code for the Local Transmission System Operator, "Arrêté du Gouvernement wallon relatif à la révision du règlement technique pour la gestion des réseaux de distribution d'électricité en Région wallonne et l'accès à ceux-ci," Walloon Energy Commission (Commission Wallone pour l' Energie-CWaPE),Wallonia, Belgium, May 2007.

[15] E.ON Netz GmbH, "Grid code-high and extra high voltage," Bayreut, Germany, April 2006.

[16] E.ON Netz GmbH, "Requirements for offshore grid connections in the E.ON Netz Network," E.ON Netz GmbH, Bayreuth, Germany, April 2008.

[17] Regulation TF 3.2.6, "Grid connection of wind turbines to networks with voltages below $100 \mathrm{kV}$," Energinet, Denmark, May 2004.

[18] V. Galdi, A. Piccolo, and P. Siano, "Exploiting maximum energy from variable speed wind power generation systems by using an adaptive Takagi-Sugeno-Kang fuzzy model," Energy Conversion and Management, vol. 50, no. 2, pp. 413-421, 2009.

[19] V. Calderaro, V. Galdi, A. Piccolo, and P. Siano, "A fuzzy controller for maximum energy extraction from variable speed wind power generation systems," Electric Power Systems Research, vol. 78, no. 6, pp. 1109-1118, 2008.

[20] A. J. Pujante-Lopez, E. Gomez-Lazaro, J. A. Fuentes-Moreno, A. Molina-Garca, and A. Vigueras-Rodriguez, "Performance comparison of a 2 MW DFIG wind turbine model under wind speed variations," in Proceedings of the Europe's Premier Wind Energy Event (Ewec'09), pp. 1-6, 2009.

[21] J. M. Carrasco, L. G. Franquelo, J. T. Bialasiewicz et al., "Powerelectronic systems for the grid integration of renewable energy sources: a survey," IEEE Transactions on Industrial Electronics, vol. 53, no. 4, pp. 1002-1016, 2006.

[22] A. Tapia, G. Tapia, J. Xabier Ostolaza, and J. R. Sáenz, "Modeling and control of a wind turbine driven doubly fed induction generator," IEEE Transactions on Energy Conversion, vol. 18, no. 2, pp. 194-204, 2003.

[23] V. Galdi, A. Piccolo, and P. Siano, "Designing an adaptive fuzzy controller for maximum wind energy extraction," IEEE Transactions on Energy Conversion, vol. 23, no. 2, pp. 559-569, 2008.

[24] http://www.winddata.com/.

[25] Mathwork, "SimPower system Toolbox of MATLAB," Mathwork, 2009. 

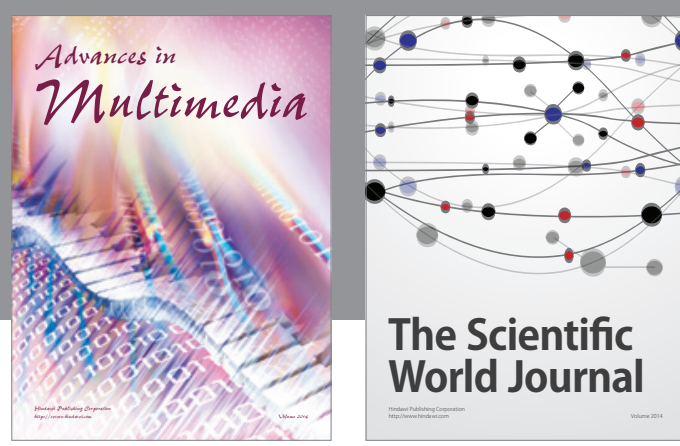

The Scientific World Journal
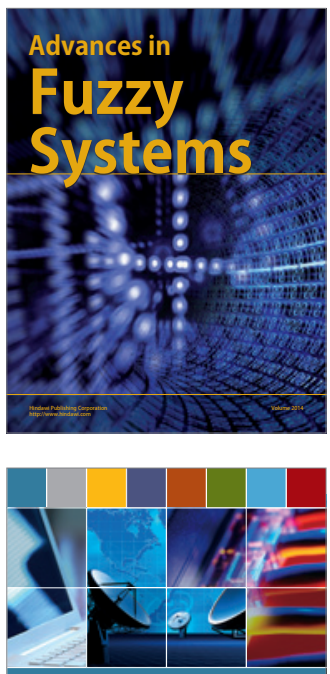

Computer Networks and Communications
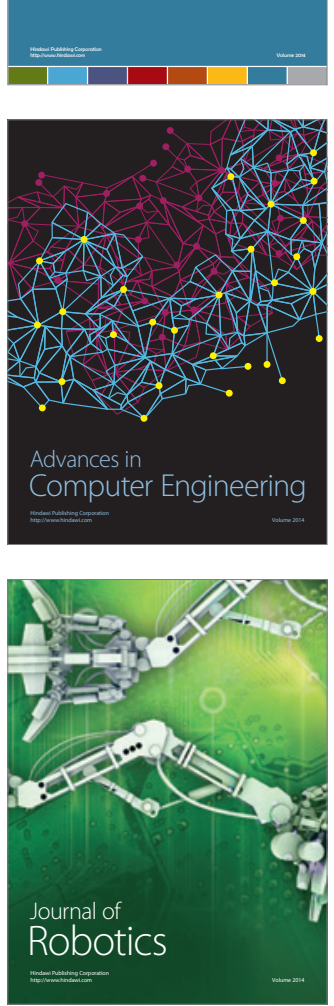
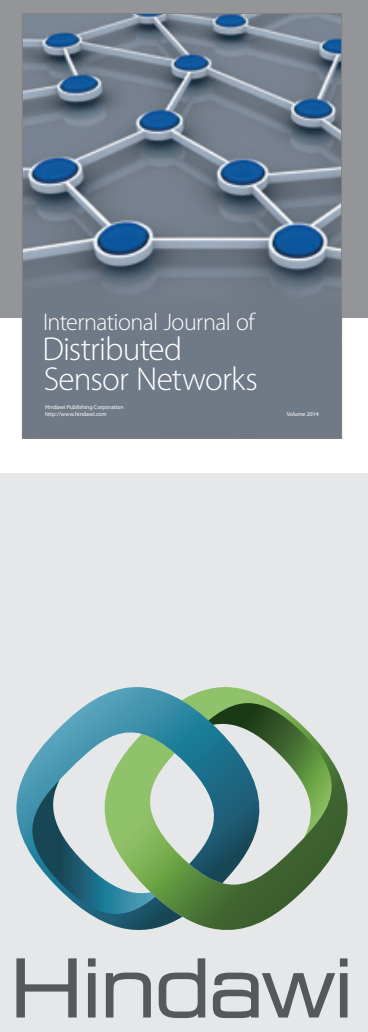

Submit your manuscripts at

http://www.hindawi.com
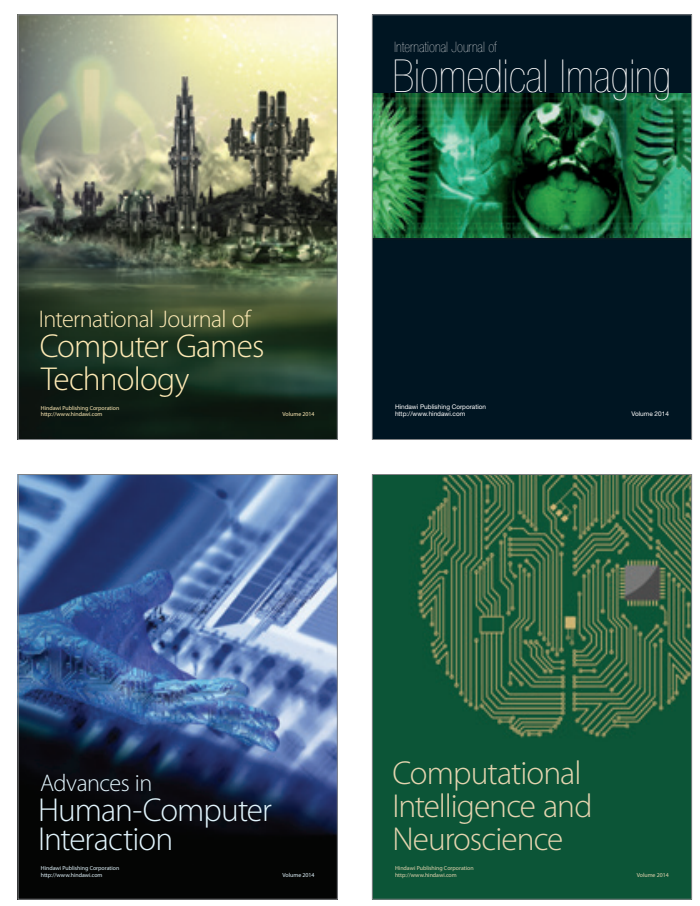
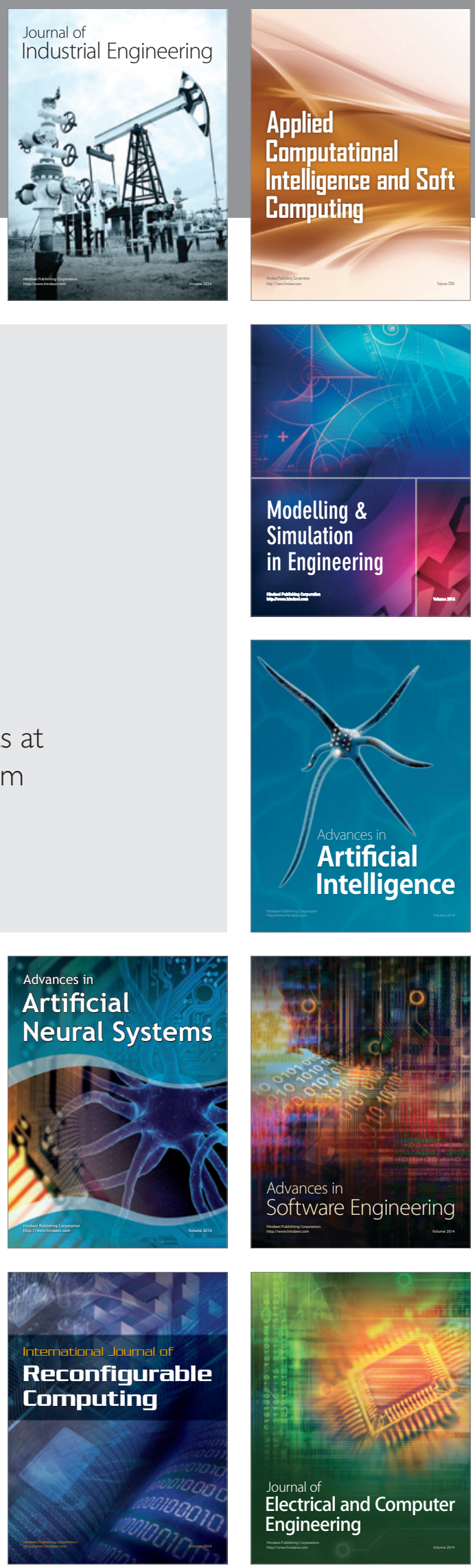